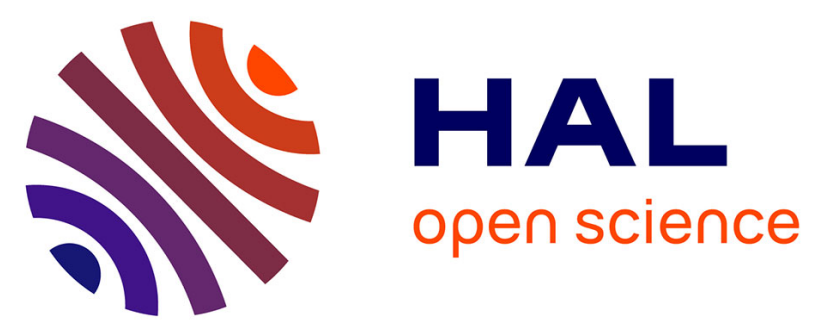

\title{
Thermodynamic properties of the Mie n-6 fluid: a comparison between Statistical Associating Fluid Theory of Variable Range (SAFT-VR) approach and Molecular Dynamics results.
}

\author{
Guillaume Galliero, Thomas Lafitte, David Bessieres, Christian Boned
}

\section{To cite this version:}

Guillaume Galliero, Thomas Lafitte, David Bessieres, Christian Boned. Thermodynamic properties of the Mie n-6 fluid: a comparison between Statistical Associating Fluid Theory of Variable Range (SAFT-VR) approach and Molecular Dynamics results.. Journal of Chemical Physics, 2007, 127, pp.184506. 10.1063/1.2801997 . hal-00322163

\section{HAL Id: hal-00322163 \\ https://hal.science/hal-00322163}

Submitted on 16 Sep 2008

HAL is a multi-disciplinary open access archive for the deposit and dissemination of scientific research documents, whether they are published or not. The documents may come from teaching and research institutions in France or abroad, or from public or private research centers.
L'archive ouverte pluridisciplinaire HAL, est destinée au dépôt et à la diffusion de documents scientifiques de niveau recherche, publiés ou non, émanant des établissements d'enseignement et de recherche français ou étrangers, des laboratoires publics ou privés. 


\title{
Thermodynamic properties of the Mie $n-6$ fluid: a
}

\section{comparison between Statistical Associating Fluid Theory}

\section{of Variable Range (SAFT-VR) approach and Molecular}

\section{Dynamics results.}

\author{
Guillaume Galliero ${ }^{1,2 *}$, Thomas Lafitte ${ }^{2}$, David Bessieres ${ }^{2}$, Christian Boned ${ }^{2}$. \\ ${ }^{1}$ Laboratoire d'Etude des Transferts d'Energie et de Matière, EA 2546, Université de Marne- \\ la-Vallée, Bâtiment Lavoisier, Champs sur Marne, F-77454 Marne la Vallée Cedex, France. \\ ${ }^{2}$ Laboratoire des Fluides Complexes, Faculté des Sciences et Techniques, UMR CNRS 5150, \\ Université de Pau, BP 1155, 64013 Pau Cedex, France. \\ *Corresponding author : E-mail : galliero@enscpb.fr ; \\ Tel : +33 5594076 88; Fax : +33 559407695
}

\begin{abstract}
Molecular Dynamics (MD) simulations of direct and derivative thermodynamic properties of the Mie $n-6$ fluid ( $n=8,10$ and 12) have been performed for liquid to supercritical states. Using the results, has been carried out an in depth test of the monomer-monomer interaction estimation of a recently derived SAFT-VR equation of state (Lafitte et al. J. Chem. Phys., 2006, 124, 024509) based on the Mie $n-6$ potential. For pure fluids, using an appropriate scaling, MD simulations show that density and isometric heat capacity are nearly independent of $n$ whereas sound velocity and thermal pressure coefficient tend to increase with $n$. In addition, the results show that predictions provided by the equation of state are consistent with those coming from MD and catch correctly the trends of each property with $n$ except for the heat capacity. The comparison is next extended to binary mixtures, with components differing
\end{abstract}


only in the value of the $n$-parameter, and demonstrates the reliability of the scheme (MX1b) used by Lafitte et al. to deal with this parameter in the SAFT-VR equation of state. In addition, is proposed a new empirical one-fluid approximation on the $n$-parameter thanks to MD simulations, which very favorably compares to one fluid model on $n$ previously proposed in the literature. The consistency of this approximation is addressed by making use of it in combination with the SAFT-VR Mie EOS. It is shown that using such an approach, which is easier to handle than the MX1b one, yields slightly improved results compared to the MX1b ones. 


\section{Introduction}

The choice of adequate analytical intermolecular potential models is still a major challenge in order to accurately describe the behavior of dense fluids. In this context, the effective two parameters Lennard-Jones 12-6 (LJ) potential is the most widely used model for exploring the behavior of simple fluids (i.e. molecules for which the most important intermolecular forces are repulsion and van der Waals dispersion) in statistical physics and related scientific domains. This potential, despite its simplicity, mimics most of the features found experimentally in fluid states. However, from a theoretical point of view, it is well known that the $\mathrm{LJ}$ potential is not a true representation of even two-body interactions between argon atoms. In particular, it represents the decay of repulsive interaction by an inversetwelve power dependence on intermolecular separation which was chosen mainly for mathematical convenience and has no physical soundness ${ }^{1}$. This apparent weakness is of importance since it appears clearly that the structural properties of a simple fluid are primarily determined by the intermolecular short-range repulsive interactions ${ }^{2,3}$. Therefore, one way to improve the results, using simple effective potentials, could be the use of different forms of the repulsive part of the potential as done for transport properties in low density gases ${ }^{4}$. In this work, among the possible simple alternatives to the two parameters LJ 12-6 potential, we have chosen the three parameters Mie $n-6$ potential family that uses a $1 / r^{n}$ formulation of the repulsive part of potential (where $r$ is the distance between particles). It should be mentioned that, recently, using molecular simulations, some interesting results on phase equilibria as well on transport properties have been obtained for this family of potential ${ }^{5-10}$.

Apart from their direct use in molecular simulations, simple effective potentials can be used as the elementary bricks of modern molecular-based Equation Of State (EOS). Perhaps the most successful of such a molecular-based EOS applicable to a large class of fluids (not restricted to simple fluids) is the Statistical Associating Fluid Theory (SAFT) approach 
proposed by Chapman et al. ${ }^{11,12}$ on the basis of Wertheim's thermodynamic perturbation theory ${ }^{13-16}$. In this context, it is important to underline that while the important feature of this approach is that it explicitly takes into account non-sphericity and association effects, one of the crucial aspect remains the accurate modeling of the repulsion and the van der Waals interactions between the monomer segments constituting the chain molecule. In fact, a wide variety of choice has been already proposed in the literature ${ }^{17-19}$. However, it has been recently showed by Lafitte et al. ${ }^{20}$ that significant discrepancies between these models arise when they are used to predict second-order derivative properties (isobaric heat capacity, isobaric thermal expansivity, speed of sound etc). Thus, making the assumption that the weakness was mainly due to the way repulsive interactions were modeled, they proposed a modification in the original SAFT-VR equation in order to deal with the Mie $n-6$ potential (the SAFT-VR Mie) for describing the interaction between the monomer forming the chain. In particular, it has been shown significant improvements for both n-alkanes ${ }^{20}$ and alcohols ${ }^{21}$ using this EOS for the estimation of all volumetric and derivative properties over wide pressure and temperature conditions without any deterioration of vapor-liquid coexistence curve. Moreover, they obtained a clear dependence of the parameters with the number of carbon atoms, which emphasizes the consistency of including the variable repulsive part as an extra pure component parameter. Regarding these encouraging results, it should be kept in mind that the adequacy of the equation to reproduce the properties of segments interacting through Mie $n-6$ potential has not yet been investigated, so that care must be taken when trying to transfer the value obtained for the repulsive exponent into other models.

Therefore, the main aim of this paper is to provide an in depth analysis of the accuracy of the monomer contribution estimation in this SAFT-VR Mie EOS. To this end, Molecular Dynamics (MD) simulations have been performed on the Mie $n-6$ fluid, in the microcanonical ensemble (NVE), for different values of the repulsive exponent 8, 10 and 12 (the EOS having 
been developed for $\mathrm{n} \leq 12$ ) in order to obtain results on thermodynamic properties. The properties computed are the pressure, the potential energy, the isometric heat capacity, the thermal pressure coefficient and the sound velocity. This is done for a large variety of thermodynamic states (12 states covering liquids and supercritical phases). It should be mentioned that, to the best of our knowledge, this is the first time derivative thermodynamic properties have been computed through molecular simulation for the Mie $n-6$ fluid when $n \neq 12$. The MD results are then compared with the results provided by the SAFT-VR Mie EOS, allowing a clear assessment of the physical soundness of this new version and the interest of making use of the Mie $n-6$ potential to model direct as well as derivative thermodynamic properties. Moreover, is also investigated the reliability of the scheme used in the EOS to deal with mixtures, based on the MX1b mixing rule of Galindo et al. ${ }^{22}$.

In addition, based on a previous work ${ }^{10}$ and using new Molecular Dynamics results, a new empirical one fluid approximation directly on the repulsive exponent is proposed in order to estimate direct and derivative properties in mixtures. Finally, this one fluid approximation ,which is easier to handle that the MX1b scheme (which goes beyond the one fluid approximation), and others previously proposed in the literature are applied in combination with the SAFT-VR Mie EOS.

\section{Theory}

\subsection{The Mie $n-6$ potential.}

In this work, the Mie $n-6$ potential which is the elementary brick of the SAFT VR Mie EOS, has been used to treat particle interactions. It can be expressed as, for an interaction between a particle $i$ and a particle $j$ :

$$
U_{i j}=\varepsilon_{i j}\left[\frac{6}{n_{i j}-6}\left(\frac{r_{m_{i j}}}{r_{i j}}\right)^{n_{i j}}-\frac{n_{i j}}{n_{i j}-6}\left(\frac{r_{m_{i j}}}{r_{i j}}\right)^{6}\right]
$$


where $r_{\mathrm{ij}}$ is the distance between particle $i$ and $j, \varepsilon_{i j}$ the potential depth, $r_{m_{i j}}$ the distance at which the potential is minimum and $n_{i j}$ the stiffness of the repulsive slope of the potential. This potential can be rewritten in terms of $\sigma_{i j}$, the position at which the potential is equal to zero (the "atomic diameter"):

$$
U_{i j}=C_{i j} \varepsilon_{i j}\left[\left(\frac{\sigma_{i j}}{r_{i j}}\right)^{n_{i j}}-\left(\frac{\sigma_{i j}}{r_{i j}}\right)^{6}\right]
$$

where

$$
C_{i j}=\frac{n_{i j}}{n_{i j}-6}\left(\frac{n_{i j}}{6}\right)^{\frac{6}{n_{i j}-6}} \text { and } \sigma_{i j}=\left(\frac{6}{n_{i j}}\right)^{\frac{1}{n_{i j}-6}} r_{m_{i j}}
$$

It should be noted that, in this work, we have restricted ourselves to the cases where $n_{i j} \leq 12$ for which the SAFTR-VR-Mie EOS only applies ${ }^{20}$. In addition, as the binary mixtures studied have been restricted to systems for which only $n$ differs between compounds, the energetic and the sizes parameters will be simply noted $\varepsilon$ and $\sigma$ in the following.

To perform MD simulations on this potential, a simple truncation at a cutoff radius, $r_{c}$, equal to $3.5 \sigma$ has been used. Consequently, long range contributions due to the truncation have been introduced (see section 2.2.3). It should be mentioned that no corrections were applied to avoid the discontinuity of the force ${ }^{23}$ deriving from the potential at $r_{i j}=r_{c}$.

\subsection{Molecular Dynamics}

\subsubsection{Combining rules}

When dealing with mixtures one needs a set of combining rules on the molecular parameters. In this work, as long as only $n$ differs between compounds a rule is only needed on the $n$ parameter. Two different combining rules have been used for $n$, the simple arithmetic one:

$$
n_{i j}=\frac{n_{i i}+n_{j j}}{2}
$$

and the one proposed for the SAFT-VR Mie $\mathrm{EOS}^{21}$ which reduces to: 


$$
n_{i j}^{3}=3+\left[\left(n_{i i}^{3}-3\right)\left(n_{j j}^{3}-3\right)\right]^{\frac{1}{2}}
$$

for the case where $\sigma_{i i}=\sigma_{j j}$.

\subsubsection{Dimensionless and reduced units:}

As this work deals only with monomer-monomer interaction (spheres), it is convenient to use dimensionless thermodynamic variables ${ }^{24}$, which are for the potential energy (the configurational internal energy):

$$
u^{*}=\frac{\sum_{i} \sum_{j>i} U_{i j}}{N \varepsilon}
$$

the temperature:

$$
T^{*}=\frac{k_{B} T}{\varepsilon}
$$

the density,

$$
\rho^{*}=\frac{N \sigma^{3}}{V}
$$

and the pressure

$$
P^{*}=P \frac{\sigma^{3}}{\varepsilon}
$$

where $k_{B}$ is the Boltzmann constant, $T$ is the temperature, $N$ the number of particles, $V$ the volume of the simulation box. All dimensionless quantities will be noted with a star as a superscript in the following.

It should be mentioned that the link between $\rho^{*}, T^{*}$ and $P^{*}$ is unique for a given potential and so differs between Mie $n-6$ potentials for different $n$, even if similarities exist ${ }^{10}$. So, in order to perform a consistent comparison between results for different $n$, the classical thermodynamic reduced conditions have been used. They are defined as:

$$
T_{r}=\frac{T^{*}}{T_{c}^{*}}, \rho_{r}=\frac{\rho^{*}}{\rho_{c}^{*}} \text { and } P_{r}=\frac{P^{*}}{P_{c}^{*}}
$$


where $T_{\mathrm{c}}{ }^{*}$ is the dimensionless critical temperature, $\rho_{\mathrm{c}}{ }^{*}$ the dimensionless critical density and $P_{\mathrm{c}}{ }^{*}$ the dimensionless critical pressure which depend $d^{5,6}$ on $n$. For these critical dimensionless properties, we have used the data of Okumura et al. ${ }^{6}$ which are summarized in table I. By using such scaling, and assuming it as adequate, a given set of $T_{r}$ and $P_{r}$ will correspond approximately to the same physical state (relatively to the critical point) whatever the potential and its repulsive coefficient.

To provide results in real units, methane has been chosen as the reference compound. We have taken $\sigma=3.7332 \AA$ and $\varepsilon=1265.3 \mathrm{~J} / \mathrm{mol}^{20}$ for the case where $n=12$. The scaling described above implies that $\sigma=3.7136 \AA$ and $\varepsilon=1112.01 \mathrm{~J}^{\mathrm{mol}}{ }^{-1}$ for $n=10$ and $\sigma=3.7137 \AA$ and $\varepsilon=915.84 \mathrm{~J} \cdot \mathrm{mol}^{-1}$ for $n=8$. These parameters have been used except when stated.

\subsubsection{Thermodynamic properties}

Concerning the configurational internal energy (potential energy), $u$, and the pressure, $P$, the usual MD formulations have been applied ${ }^{23}$. In addition, due to the use of truncated Mie $n-6$ potential, long-range corrections noted with a subscript $l r$, have been included for pressure and for potential energy estimation. Using the general formulations ${ }^{25}$, after a few calculations is obtained:

$$
\begin{aligned}
& u_{l r}^{*}=2 \pi \rho^{*}\left[\frac{n}{n-6}\left(\frac{n}{6}\right)^{\frac{6}{n-6}}\left(\frac{1}{n-3}\left(\frac{1}{r_{\text {cut }}^{*}}\right)^{n-3}-\frac{1}{3}\left(\frac{1}{r_{\text {cut }}^{*}}\right)^{3}\right)\right] \\
& P_{l r}^{*}=\frac{2}{3} \pi \rho^{* 2}\left[\frac{n}{n-6}\left(\frac{n}{6}\right)^{\frac{6}{n-6}}\left(\frac{n}{n-3}\left(\frac{1}{r_{\text {cut }}^{*}}\right)^{n-3}-2\left(\frac{1}{r_{\text {cut }}^{*}}\right)^{3}\right)\right]
\end{aligned}
$$

where $r_{\text {cut }}^{*}$ is the dimensionless cutoff radius (equal to 3.5 in the simulations).

Concerning the second-derivative properties (thermodynamic response functions), it exists several ways to obtain them from molecular simulations ${ }^{25}$. The most accurate is the one based on statistical mechanics that involves the measure of fluctuations. In the microcanonical 
ensemble (NVE), in which the simulations data have been collected, the dimensionless isometric heat capacity is expressed $\mathrm{as}^{25}$ :

$$
C_{V}^{*}=\frac{C_{V}}{N k_{B}}=\left[N-N T^{*}\left(\frac{3 N}{2}-1\right)\left\langle E_{k}^{*-1}\right\rangle\right]^{-1}
$$

where $E_{k}$ is the kinetic energy.

The dimensionless thermal pressure coefficient, writes as ${ }^{25}$ :

$$
\gamma_{v}^{*}=\frac{\gamma_{v} \sigma^{3}}{k_{B}}=\frac{2}{3} C_{v}^{*}\left[\rho^{*}-\frac{1}{T^{* 2}}\left\langle\delta E_{k}^{*} \delta P^{*}\right\rangle\right]
$$

where

$$
\left\langle\delta E_{k}^{*} \delta P^{*}\right\rangle=\left\langle\left(E_{k}^{*}-\left\langle E_{k}^{*}\right\rangle\right)\left(P^{*}-\left\langle P^{*}\right\rangle\right)\right\rangle
$$

The adiabatic compressibility can be expressed $\mathrm{as}^{25}$ :

$$
\kappa_{s}=\left[\frac{2}{3} P+\rho k_{B} T+\langle\theta\rangle-\frac{N}{\rho k_{B} T}\left\langle(\delta P)^{2}\right\rangle\right]^{-1}
$$

where:

$$
\theta=\frac{1}{9 V} \sum_{i} \sum_{i<j} r_{i j}^{2} \frac{\partial^{2} U_{i j}}{\partial r_{i j}^{2}}
$$

After some calculations, the dimensionless adiabatic compressibility can be rewritten for a Mie $n-6$ potential as:

$$
\kappa_{s}^{*}=\frac{\kappa_{s} \varepsilon}{\sigma^{3}}=\left[\frac{2-a}{3} P^{*}+\frac{3+a}{3} \rho^{*} T^{*}+\frac{b}{9} \rho^{*} u^{*}-\frac{N}{\rho^{*} T^{*}}\left\langle\left(\delta P^{*}\right)^{2}\right\rangle\right]^{-1}
$$

with

$$
a=\frac{n(n+1)-42}{6-n}, b=42+6 a
$$

The sonic velocity $\omega^{*}$ is deduced from $\kappa_{\mathrm{s}}{ }^{*}$ using the classical macroscopic thermodynamic relation: 


$$
\omega^{*}=\left(\rho^{*} \kappa_{s}^{*}\right)^{-\frac{1}{2}}
$$

The other derivative thermodynamic properties can be deduced from the previous ones using classical macroscopic thermodynamic relations.

\subsection{SAFT-VR Mie Equation of state.}

The residual Helmholtz free energy $\left(A^{R E S}\right)$ of a mixture of associating chain molecules can be written as the addition of three contributions (monomer, chain and association) as:

$$
\frac{A^{R E S}}{N k_{B} T}=\frac{A^{M O N O}}{N k_{B} T}+\frac{A^{\text {CHAIN }}}{N k_{B} T}+\frac{A^{A S S O C}}{N k_{B} T}
$$

Note that since we are treating non-associating spherical segments, the chain and the association contribution to the free energy are not considered here. Therefore the expression for the residual Helmholtz free energy is reduced to the monomer term which is obtained from the Barker-Henderson high-temperature expansion for hard-core systems up to second order, i.e.

$$
a^{R E S}=a^{H S}+\beta a_{1}^{M}+\beta^{2} a_{2}^{M}
$$

where we define $a=A / N k_{B} T$ as the reduced Helmholtz free energy per molecule. In this expression $a^{H S}$ represents the hard-sphere free energy of the mixture, and $a_{1}{ }^{M}$ and $a_{2}^{M}$ are the first two terms associated with the attractive part of the Mie potential.

The expression of Boublik ${ }^{26}$ and Mansoori et al..$^{27}$ is used for the reference hard-sphere term:

$$
a^{H S}=\frac{6}{\pi \rho_{s}}\left[\left(\frac{\zeta_{2}^{3}}{\zeta_{3}^{2}}-\zeta_{0}\right) \ln \left(1-\zeta_{3}\right)+\frac{3 \zeta_{1} \zeta_{2}}{1-\zeta_{3}}+\frac{\zeta_{2}^{3}}{\zeta_{3}\left(1-\zeta_{3}\right)^{2}}\right]
$$

where $\rho_{s}$ is the number density of spherical segments. The packing fraction $\zeta_{n}$ is defined by:

$$
\zeta_{n}=\frac{\pi \rho_{s}}{6}\left[\sum_{i} x_{i} \sigma_{i i}^{B H^{n}}\right]
$$


$\sigma_{i i}^{B H}$ represents the hard-core temperature dependent diameter of the segments in the molecule $i$. Note that $x_{i}$ is the mole fraction of type $i$ segments in the system. The expression for the evaluation of the temperature dependent diameter is given by Barker and Henderson ${ }^{28}$ :

$$
\sigma_{i i}^{B H}(T)=\int_{0}^{\sigma_{i}}\left(1-\exp \left(\frac{-u_{i i}^{M}}{k T}\right)\right) d r
$$

The mean-attractive term $a_{1}{ }^{M}$ of the mixture is the sum of the terms for each type of pair interaction $^{22}$ :

$$
a_{1}^{M}=\sum_{i}^{n} \sum_{j}^{n} x_{i} x_{j} a_{1}^{i j}
$$

The analytical expression for $a_{1}^{i j}$ for monomer segments interacting through Mie $n-6$ potential can be written as a sum of two Sutherland perturbation terms:

$$
a_{1}^{i j}=C_{i j}\left[-a_{1}^{s, i j}\left(\lambda_{i j}=n_{i j}\right)+a_{1}^{s, i j}\left(\lambda_{i j}=6\right)\right]
$$

In the original paper on SAFT-VR, Gil-Villegas et al. ${ }^{17}$ showed that the mean-attractive term $a_{1}^{s, i j}$ could be expressed in terms of the contact value of $g_{i j}^{H S}$ and an effective packing fraction $\zeta_{3}^{\text {eff }}$ :

$$
a_{1}^{s, i j}\left(\lambda_{i j}\right)=-\rho_{s} \alpha_{i j}^{V D W}\left(\lambda_{i j}\right) g_{i j}^{H S}\left[\sigma_{i j}^{B H} ; \zeta_{3}^{e f f}\left(\lambda_{i j}\right)\right]
$$

In this expression $\alpha_{i j}^{V D W}$ represents the van der Waals mean-field term for Sutherland fluids evaluated with the temperature dependent diameter $\sigma_{i j}^{B H}$ :

$$
\alpha_{i j}^{V D W}\left(\lambda_{i j}\right)=2 \pi \varepsilon_{i j} \sigma_{i j}^{B H^{3}} \frac{1}{\lambda_{i j}-3}
$$

Note that $\sigma_{i j}^{B H}$ is the distance between centers of segment $i$ and $j$ at contact. It is given by the Lorentz Bethelot combining rule:

$$
\sigma_{i j}^{B H}=\frac{\sigma_{i i}^{B H}+\sigma_{j j}^{B H}}{2}
$$


In this work, we have decided to use the mixing rule MX1b as defined in Gil-Villegas et al.17. Indeed, Galindo et al. ${ }^{22}$ showed that this approach is more convenient for the study of the global phase behavior, if compared to higher order approximation, while keeping the same degree of accuracy away from the critical region.

In this context, the van der Waals one fluid theory is used to approximate the value of $g_{i j}^{H S}$. Consequently, the radial distribution function for a hypothetical pure fluid $g_{0}^{H S}$ of average diameter $\sigma_{x}^{B H}$ is incorporated in equation (28) as:

$$
a_{1}^{s, i j}\left(\lambda_{i j}\right)=-\rho_{s} \alpha_{i j}^{V D W}\left(\lambda_{i j}\right) g_{0}^{H S}\left[\sigma_{x}^{B H} ; \zeta_{3}^{e f f}\left(\lambda_{i j}\right)\right]
$$

where $\zeta_{x}^{\text {eff }}$ stands for an effective packing fraction ${ }^{22}$ related to the packing fraction of the mixture $\zeta_{x}$. The effective packing fraction $\zeta_{x}^{\text {eff }}$ is approximated from:

$$
\zeta_{x}^{e f f}\left(\lambda_{i j}\right)=c_{1}\left(\lambda_{i j}\right) \zeta_{x}+c_{2}\left(\lambda_{i j}\right) \zeta_{x}^{2}
$$

where

$$
\zeta_{x}=\frac{\pi}{6} \rho_{s} \sigma_{x}^{B H^{3}}
$$

with

$$
\sigma_{x}^{B H}=\sum_{i} \sum_{j} x_{i} x_{j} \sigma_{i j}^{B H^{3}}
$$

The coefficients $c_{n}$, which are functions of $\lambda_{i j}$, are given by the matrix ${ }^{17}$ :

$$
\left(\begin{array}{l}
c_{1} \\
c_{2}
\end{array}\right)=\left(\begin{array}{cccc}
-0.943973 & 0.422543 & -0.0371763 & 0.00116901 \\
0.370942 & -0.173333 & 0.0175599 & -0.000572729
\end{array}\right)\left(\begin{array}{l}
1 \\
\lambda_{i j} \\
\lambda_{i j}^{2} \\
\lambda_{i j}^{3}
\end{array}\right)
$$

The radial distribution function at contact $g_{0}^{H S}\left(\sigma_{x}^{B H}, \zeta_{x}^{e f f}\right)$ is obtained from the Carnahan and Starling ${ }^{29}$ equation of state as follows:

$$
g_{0}^{H S}\left[\sigma_{x}^{B H} ; \zeta_{x}^{e f f}\right]=\frac{1-\zeta_{x}^{e f f} / 2}{\left(1-\zeta_{x}^{e f f}\right)^{3}}
$$


The second perturbation term $a_{2}^{M}$ is given by :

$$
a_{2}^{M}=\sum_{i}^{n} \sum_{j}^{n} x_{i} x_{j} a_{2}^{i j}
$$

where $a_{2}^{i j}$ is related to $a_{1}^{i j}$ as follows :

$$
a_{2}^{i j}=\frac{C_{i j}}{2} \varepsilon_{i j} K^{H S} \rho_{s} \frac{\partial a_{1}^{s}\left(\lambda_{i j}=12\right)}{\partial \rho_{s}}
$$

In this expression $K^{H S}$ stands for the Percus-Yevick ${ }^{30}$ expression for the isothermal compressibility of a hard-sphere fluid,

$$
K^{H S}=\frac{\zeta_{0}\left(1-\zeta_{3}\right)^{4}}{\zeta_{0}\left(1-\zeta_{3}\right)^{2}+6 \zeta_{1} \zeta_{2}\left(1-\zeta_{3}\right)+9 \zeta_{2}^{3}}
$$

Finally, it should be highlighted that the EOS with the present formulation for mixtures, the so-called MX1b mixing rule, turns out to be a combination of two approaches: the two fluids level which is of interest to maintain a good accuracy for the description of the structure of the fluid, and the simpler one fluid approximation which is used to derive the dispersive term in order to avoid inconsistencies in calculations close to the critical state.

However, it is important to note that the simplest van der Waals one fluid approximation could be used at all levels in the EOS with the appropriately chosen molecular parameters to represent the "equivalent" pseudocompound, see section 2.4. A comparison between these two schemes is provided in section 4.2.2.

\subsection{One-fluid approximation}

The classical way to deal with thermophysical properties of fluid mixture in the liquid state community is to treat the mixture as a "pure" fluid by defining a pseudo compound "equivalent" to the mixture through a one-fluid approximation ${ }^{31-32}$. At the microscopic scale, for the LJ fluid, such formulation corresponds to the definition of the molecular parameters of the pseudo compound, $\sigma_{1-f l u i d}, \varepsilon_{1-f l u i d}$, starting from those of the pure compounds and those 
defined through the combining rules. One aim of this work is to define to which extent such an approach can be efficient concerning the $n$ parameter for the Mie potential (i.e. how to define $n_{1-f l u i d}$ in binary mixtures where only the repulsive stiffness $n$ differs between compounds). To achieve such a goal, we have tested various one-fluid models on $n$ :

the linear one:

$$
n_{1-\text { fluid }}=\sum_{i} \sum_{j} x_{i} x_{j} n_{i j}
$$

one proposed in ${ }^{22}$ :

$$
n_{1-\text { fluid }}^{3}=\sum_{i} \sum_{j} x_{i} x_{j} n_{i j}^{3}
$$

one related to a previous work ${ }^{10}$ :

$$
\left(n_{1-f l u i d}-6\right)^{\frac{1}{2}}=\sum_{i} \sum_{j} x_{i} x_{j}\left(n_{i j}-6\right)^{\frac{1}{2}}
$$

and two new empirical ones:

$$
\begin{gathered}
\frac{n_{1-\text { fluid }}-6}{n_{1-\text { fluid }}}=\sum_{i} \sum_{j} x_{i} x_{j} \frac{n_{i j}-6}{n_{i j}} \\
\left(\frac{n_{1-\text { fluid }}-6}{n_{1-\text { fluid }}}\right)^{\frac{1}{2}}=\sum_{i} \sum_{j} x_{i} x_{j}\left(\frac{n_{i j}-6}{n_{i j}}\right)^{\frac{1}{2}}
\end{gathered}
$$

where $x_{i}$ is the molar fraction of component $i$.

\section{Simulations details}

\subsection{Technical details.}

Simulations have been performed using a homemade code on systems composed of 1000 particles. To integrate the equation of motion a Velocity Verlet algorithm has been applied with a dimensionless timestep of 0.002 . To save CPU time, a Verlet neighbour list combined with a cell list ${ }^{23}$ has been used. Classical periodical boundary conditions were applied. 
To obtain the thermophysical properties in the desired state, simulations have been performed in two steps: first the system has been driven towards the desired temperature and pressure, using Berendsen thermostat and barostat (NPT simulations) ${ }^{33}$ during $10^{5}$ timesteps (with a coupling constant equal to $10^{3}$ timesteps for the pressure and $510^{2}$ timesteps for the temperature). Then, starting from this configuration, data have been collected during 1.5-3 10 timesteps of NVE simulations to compute the thermodynamic properties using the relations provided in section 2.2.3. Data obtained during the NPT were discarded from the analysis. With these parameters, during the NVE simulations, the estimated errors (using the subblocks average method ${ }^{23}$ with subblock composed of $1.510^{5}$ timesteps to ensure the statistical independence) are around $0.1-0.2 \%$ for $T$ and $u$ (and so omitted in the Appendix), below $1 \%$ for $P$, below $3 \%$ for the isometric heat capacity and the sound velocity and around $3-4 \%$ for the thermal pressure coefficient, see Appendix A and C.

To carry out a consistent comparison between MD and SAFT-VR results, first the MD simulations are performed to obtain all thermodynamic properties during NVE runs. Second, the MD values obtained for $T$ and $P$ are used as input parameters for the EOS which is then used to predict the thermodynamic properties $\left(u, \rho, \omega, \gamma_{v}\right.$ and $\left.C_{v}\right)$ and allows the comparison on these properties.

\subsection{Validation.}

To assess the reliability of our MD results (in complement to the comparison with the EOS of Kolafa and Nezbeda ${ }^{34}$ already done in Galliero et al. ${ }^{35}$ ), simulations have been performed on a LJ pure fluid for which Meier ${ }^{36}$ provides recent accurate data on thermodynamic properties. The states chosen, $\rho^{*}=0.75$ to 0.9 with a step of 0.5 at $T^{*}=1$ and $\rho^{*}=0.1$ to 1 with a step of 0.1 at $T^{*}=2.5$, cover the range of thermodynamics conditions tested in this work. The deviations, Dev, have been evaluated thanks to:

$$
\operatorname{Dev}=\left(\chi_{\text {this_work }}-\chi_{\text {Meier }}\right) / \chi_{\text {Meier }}
$$


where $\chi_{\text {this_work }}$ represents our molecular dynamics value on the variable $\chi$ and $\chi_{\text {Meier }}$ the Meier's ${ }^{36}$ one. The Absolute Average Deviation (AAD), for the $N B$ values of deviation, is defined by:

$$
\mathrm{AAD}=\frac{1}{N B} \sum_{i=1}^{N B}|\operatorname{Dev}(i)|
$$

the maximum deviation by (DMax):

$$
\operatorname{DMax}=\max (|\operatorname{Dev}(i)|)
$$

and the bias by:

$$
\operatorname{Bias}=\frac{1}{N B} \sum_{i=1}^{N B} \operatorname{Dev}(i)
$$

Results provided in table II indicate that our computations are consistent with those of Meier the deviations being always small (within the error bars). This indicates that thermodynamics properties in such monophasic conditions are not strongly affected by the cutoff radius (when long range corrections are included), as Meier uses a $5 \sigma$ cutoff whereas ours is equal to $3.5 \sigma$. In addition, it should be mentioned that deviations are, by part, due to the fact that our computations are performed in the NVE ensemble and not the NVT one (in this case the AAD on temperature is of $0.2 \%$ ).

\section{Results}

\subsection{Comparison between MD and EOS results}

\subsubsection{Pure fluids}

The main aim of this work is to quantify to which extent the SAFT-VR-Mie EOS is able to reproduce molecular simulation data of the Mie $n-6$ pure fluid, both concerning direct and derivative thermodynamic properties. Three different values of $n$ have been tested, $n=8,10$ and 12 , covering the usual range found for this parameter using the $\operatorname{EOS}^{20}$. For each $n, 12$ state points have been simulated and the results compared with what provided by the EOS. To 
perform a consistent comparison between results for various $n$, the simulations have been performed for the same set (for each $n$ ) of reduced temperature and pressure. Nevertheless, due to the fact that the properties computation were performed during $N V E$ simulations and not NPT ones, see section 3.1, $T_{r}$ and $P_{r}$ could slightly vary for different $n$. These points correspond to four different isotherms very close to, $T_{r}=0.65,0.75,0.85$ and $1.5(130,150$, 170 and $300 \mathrm{~K}$ for methane) and three isobars very close to $P_{r}=1.98,13.86$ and $29.7(10,70$, and $150 \mathrm{MPa}$ for methane). State 1 will denote the state for which $T_{r}=0.65$ and $P_{r}=1.98$, state $2 T_{r}=0.65$ and $P_{r}=13.86$ and so on up to state $12, T_{r}=1.5$ and $P_{r}=29.7$. Thus, states 1 to 9 are subcritical (liquid) and states 10 to 12 are supercritical. All molecular dynamics results are provided in Appendix A and all EOS ones in Appendix B.

In tables III and IV, deviations have been computed using:

$$
\operatorname{Dev}=\left(\chi_{E O S}-\chi_{M D}\right) / \chi_{M D}
$$

where $\chi_{M D}$ represents the molecular dynamics value of the variable $\chi$ and $\chi_{E O S}$ its predicted value using the SAFT-VR Mie EOS. AAD, DMax and Bias have been defined through equations (46-48).

Concerning the efficiency of the EOS, table III shows that despite differences, results on direct and derivative properties provided by the EOS are consistent with those coming from MD compared to the fact that no adjustable parameters have been introduced. Results are somewhat better for $\rho^{*}$ and $u^{*}$ and are globally of a similar accuracy for the three derivative properties. It should be noted that the largest deviations on $\rho^{*}$ and $u^{*}$ have been obtained for the less dense system (state 10).

In addition, generally, the EOS underestimates $\omega^{*}$ and $\gamma_{v}{ }^{*}$ and overestimates $C_{v}{ }^{*}$. Furthermore concerning the trends with $n$, table III shows that the prediction of the EOS on $\omega^{*}$ and $\gamma_{v}^{*}$ are not affected by the repulsive exponent whereas the prediction on $C_{v}{ }^{*}$, and to less extent $u^{*}$ and $\rho^{*}$, tends to slightly deteriorate with the decrease of $n$. 
Another interesting point is to compare how the thermodynamic properties, provided by MD and the EOS, evolve with $n$ for the different states studied. Such trend is shown on figures 1-2 where the deviations shown correspond to:

$$
\operatorname{Dev}=\left(\chi_{M i e}-\chi_{L J}\right) / \chi_{L J}
$$

where $\chi_{\text {Mie }}$ represents the value of the variable $\chi$ for the Mie potential ( $n=8$ or 10 )and $\chi_{L J}$ the value for the LJ potential $(n=12)$. Both values, $\chi_{\text {Mie }}$ and $\chi_{L J}$, have been expressed in real units for this comparison.

Molecular dynamics results show that, see figure 1.a, $\rho$ is weakly dependent on $n$, this behavior being correctly reproduced by the EOS, figure 1.b. Thus, due to this weak dependence on $n$, an adjustment of the $n$ parameter on $\rho$ alone will not ensure a clear discrimination of the "adequate" $n$ value when applied on real substance.

Concerning derivative properties, MD simulations indicate that $\omega$ as well as $\gamma_{\mathrm{v}}$ are the lowest for the Mie 8-6 potential and tend to increase with $n$ (this increase being more important at low T), see figure 2.a. Furthermore, the EOS correctly takes this behavior with $n$ into account see figure 2.b. It should be noted that such a dependence of the Mie $n-6$ fluid shows clearly the non-conformal behavior between potentials having different $n$ values. The case of the isometric heat capacity, $C_{v}$, is different, see figure 1. MD simulations yield an independent (or nearly) value with $n$, whereas the EOS predicts a slight increase; such a result explaining the trend noticed in table III. Besides, the increase of the sound velocity with $n$ can be understood in a naïve way: decreasing $n$ corresponds to a less stiff repulsion (a softer medium) between particles which should lowers the propagation of the sound as it is usually the case in solids. An interesting point, for the application of the EOS on real substances, is the fact that different $n$ parameters lead to noticeably different $\omega$ values, for a similar $\rho$, which indicates that an adjustment of the $n$ parameter on both $\rho$ and $\omega$ (as done for the SAFT-VR Mie $\operatorname{EOS}^{20}$ ) will lead unambiguously to a unique discriminative value of $n$ (at least for the monomer). 


\subsubsection{Mixtures}

Another important point is the way the SAFT-VR Mie EOS models mixtures composed of species differing only in their value of $n$. To do so, simulations on binary mixtures of particles having the same molecular parameters except $n\left(n_{11}=8, n_{22}=12\right.$ and $\sigma=3.7136 \AA$ and $\varepsilon=$ 1112.01 J.mol ${ }^{-1}$ ) have been performed. Three mole fractions have been investigated, $x_{1}=0.25$, 0.5 and 0.75 . To treat cross interactions during MD simulations, the combining rule given by equation (5) has been used as in the EOS. Similarly to the pure fluid case, twelve different states have been simulated which corresponds in real units, to $T=130,150,170$ (liquid) and $300 \mathrm{~K}$ (supercritical) combined with $P=10,70$, and $150 \mathrm{MPa}$. All molecular dynamics results are provided in dimensionless units in Appendix C and all EOS ones in Appendix D.

Table IV clearly indicates that the scheme used to model mixtures (at least concerning the $n$ parameter) in the SAFT-VR Mie EOS is efficient, as long as deviations found are of the same order as on pure fluids, see table III. Furthermore, the main evolution of the deviations with the molar fraction is the increase on $C_{v}{ }^{*}$ ones with the concentration of compound $1\left(n_{11}=8\right)$ which is consistent with what found on pure fluids.

\subsection{One-fluid approximation on the $n$ parameter}

\subsubsection{Choice of the one fluid model}

Another interesting point, even if the EOS scheme for mixture is efficient, is to determine whether or not it is possible to mimic a mixture by an "equivalent" pseudocompound with a repulsive exponent $n_{1-f l u i d}$ defined by an adequate one-fluid approximation. To define the most appropriate rule among those tested, equations (40-44), the problem has been taken in reverse: simulations have been performed on binary mixtures (for which only $n$ differs between compounds, $n_{11}=8$ and $n_{22}=12$ ) with various molar fractions and compared with those given by the $n=10$ pure fluid $\left(\sigma=3.7136 \AA\right.$ and $\left.\varepsilon=1112.01 \mathrm{~J} . m o l^{-1}\right)$. The various compositions have been deduced from the five different one fluid approximations tested, equations (40-44). 
For sake of simplicity, the linear combining rule on $n$, eq. (4), has been used. All simulations on mixtures have been performed for the same 12 states than on pure fluid using $T$ and $\rho$ as simulations input parameters (with slight deviations on $T$ as simulations have been performed in the $N V E$ ensemble and not in the $N V T$ one). For figures 3-5, deviations have been computed using

$$
\operatorname{Dev}=\left(\chi_{1-\text { fluid }}-\chi_{\text {Mix }}\right) / \chi_{\text {Mix }}
$$

where $\chi_{\text {Mix }}$ represents the MD value of the variable $\chi$ in the mixture and $\chi_{1 \text {-fluid }}$ its MD value using the one fluid model. AAD, DMax and Bias have been defined using respectively eqs (46-48).

Quite surprisingly, deviations induced by the various one fluid models on $C_{v}{ }^{*}, \omega^{*}$ and $\gamma_{v}{ }^{*}$ remain limited, below $5 \%$ and without a systematic trend, whatever the model chosen, see figure 4. On the contrary, the one fluid model may largely affects pressure, the worst results being those provided by the use of eq. (41), with $\mathrm{AAD}=16.21 \%$, and the better those induced by one of the empirical law, eq. (44), for which $\mathrm{AAD}=1.41 \%$. In addition, even if the deviations on $u^{*}$ remains limited, see figure 3 , the trends are similar to those on pressure. From these results it appears that the "usual" one fluid models, eqs (40-41), are inappropriate, at least for pressure and to a less extent to energy, and that a better choice is the one provided by eq. (44), which yields an AAD below $1.5 \%$ for all properties studied, which is excellent compared to the various uncertainties.

\subsubsection{Application of the one fluid model using MD and EOS}

In order to confirm our previous results on the one fluid model, using eq. (44), simulations have been performed on the pseudocompounds "equivalent" to the mixtures studied in section 4.1.2. In addition, the SAFT-VR Mie EOS combined with this one fluid model on $n$ has been applied to these systems. For the studied mixtures, eq. (44) leads to $n_{1-\text { fluid }}=10.65$ for $x_{1}=0.25$, $n_{1-\text { fluid }}=9.57$ for $x_{1}=0.5$ and $n_{1-\text { fluid }}=8.7$ for $x_{1}=0.75$. Simulations on the one fluid model have 
been performed so that $T$ and $P$ are the same as in the mixtures (with slight deviations on $T$ and $P$ as simulations have been performed in the $N V E$ ensemble and not in the $N P T$ one). It should be mentioned that, in this case, the combining rule defined by eq. (5) has been used as long as this rule is the one employed in the usual SAFTR-VR Mie EOS scheme on mixture. MD results shown on figure 5 are consistent with our previous findings which is that eq. (44) is an appropriate empirical one-fluid model for the parameter $n$ when dealing with Mie $n-6$ potentials. In fact, all thermodynamic properties, direct and derivative, are correctly estimated using the one fluid model whatever the state, the maximum deviation remaining below $3.5 \%$, see figure 5. This result is interesting as it shows that the choice of the combining rule (in this case eq. (5) whereas eq. (4) was used in the previous section) does not seem to affect the quality of the proposed one fluid model, which reinforce the fact that eq. (44) is adequate.

Finally, we have applied the proposed one fluid approximation on $n$, eq. (44), together with the SAFT-VR Mie EOS to see to which extent such an approach is able to provide consistent results on mixtures. In table $\mathrm{V}$, deviations have been defined by:

$$
\operatorname{Dev}=\left(\chi_{E O S-1 \text { fluid }}-\chi_{M D}\right) / \chi_{M D}
$$

where $\chi_{M D}$ represents the molecular dynamics value of the variable $\chi$ and $\chi_{\text {EOS-1fluid }}$ its predicted value using the EOS combined with the one fluid model.

Results are summarized in table V. It is interesting to note, see tables IV-V, that this scheme provides slightly better results (except for $u^{*}$ ) than those obtained using the usual scheme, the MX1b one which goes beyond a one fluid approximation on $n$. Such a result is interesting as long as a one-fluid approximation directly on the molecular parameter (here $n$ ) is more simple to handle. Nevertheless, further work should be performed in order to have a complete set of a van der Waals one-fluid approximation on the three molecular parameters, $\varepsilon, \sigma$ and $n$, which is out of the scope of this paper. 


\section{Conclusion}

In this work, an analysis of the efficiency of a recently developed SAFTR VR Mie $\operatorname{EOS}^{20}$ to provide the direct and derivative thermodynamic properties of the Mie $n-6$ pure fluid for $n$ varying from 8 to 12 has been carried on through in depth comparisons with molecular dynamics simulations in various thermodynamic states covering from liquid to supercritical states.

On pure fluids, with an appropriate scaling, MD results show that $\omega$ and $\gamma_{v}$ tend to increase with $n$ (which is an expression of the non conformal behavior between potentials having different $n$ values) whereas $\rho$ and $C_{v}$ are nearly independent of $n$. It should be noted that such behavior with $n$ is crucial for adjusting this parameter on real substance. In addition, it has been shown that the EOS is able to correctly reproduce these general trends and amplitudes of the thermodynamic properties with $n$, except concerning $C_{v}$ for which deviations may rise up to $20 \%$ (the EOS predicts a slight increase with $n$ ).

Concerning mixture, for which only the $n$ parameter varies between compounds, this EOS, combined with the previously used mixing rules MX1b, has shown to provide results as good as on pure fluid. These results unambiguously show that the monomer contribution of the SAFT-VR Mie EOS, based on the Mie $n-6$ potential, is adequately modeled by the EOS in pure fluids and mixtures, which ensures that the $n$ parameter adjustment on both $\rho$ and $\omega$ makes sense in the application of the EOS to real fluids ${ }^{20,21}$.

Elsewhere, a test of various one fluid approximations on $n$ has been achieved using MD simulations between mixtures and their "equivalent" pseudocompounds. It has been shown that the "usual" one fluid models for $n$, eqs (40-41), may induce non negligible deviations on pressure, up to $30 \%$ and $50 \%$ respectively, but small ones on other properties. To fix this weakness, following a previous work ${ }^{10}$, an empirical one-fluid approximation, eq. (44), is proposed which provides excellent results on all properties, AAD being always lower than 
$1.5 \%$. Finally, it is shown that the combination of this new one-fluid approximation on $n$ with the EOS is able to provide results on mixtures slightly improved, except on $u$ compared to those yielded by the MX1b approach (which goes beyond the one fluid approximation) and is, by far, easier to handle.

\section{Acknowledgement}

We thank the Centre Informatique National de l'Enseignement Supérieur (Montpellier, France) and the TREFLE laboratory (Bordeaux, France) which provided a large part of the computer time used for this study. 


\section{Appendix A:}

MD results on pure fluids for different $n$. Values in parenthesis represent the error on the last digits (errors on $u^{*}$ and $T^{*}$ are below $0.2 \%$ and $\rho^{*}$ is imposed).

\begin{tabular}{|c|c|c|c|c|c|c|c|}
\hline$n$ & $T^{*}$ & $\rho^{*}$ & $u^{*}$ & $P^{*}$ & $C_{v}^{*}$ & $\omega^{*}$ & $\gamma_{v}^{*}$ \\
\hline \multirow{12}{*}{12} & 0.856 & 0.7965 & -5.648 & $0.248(1)$ & $2.448(29)$ & $5.155(102)$ & $5.097(153)$ \\
\hline & 0.851 & 0.8809 & -6.141 & $1.747(3)$ & $2.690(33)$ & $6.552(123)$ & $6.774(184)$ \\
\hline & 0.852 & 0.9446 & -6.419 & $3.713(4)$ & $2.895(35)$ & $7.702(174)$ & $8.155(264)$ \\
\hline & 0.985 & 0.7411 & -5.176 & $0.256(1)$ & $2.248(25)$ & $4.599(93)$ & $3.912(138)$ \\
\hline & 0.985 & 0.8442 & -5.802 & $1.735(3)$ & $2.507(23)$ & $6.229(110)$ & $5.638(183)$ \\
\hline & 0.987 & 0.9165 & -6.138 & $3.725(5)$ & $2.727(34)$ & $7.477(155)$ & $7.032(273)$ \\
\hline & 1.120 & 0.6765 & -4.655 & $0.259(1)$ & $2.117(22)$ & $3.968(72)$ & $2.960(65)$ \\
\hline & 1.119 & 0.8081 & -5.478 & $1.728(3)$ & $2.381(27)$ & $5.902(102)$ & $4.830(185)$ \\
\hline & 1.118 & 0.8894 & -5.877 & $3.706(4)$ & $2.589(35)$ & $7.249(103)$ & $6.184(217)$ \\
\hline & 1.960 & 0.1489 & -0.988 & $0.246(1)$ & $1.651(18)$ & $1.876(29)$ & $0.198(19)$ \\
\hline & 1.977 & 0.6021 & -3.770 & $1.733(3)$ & $1.906(25)$ & $4.471(83)$ & $1.945(76)$ \\
\hline & 1.968 & 0.7408 & -4.520 & $3.706(5)$ & $2.105(26)$ & $6.200(127)$ & $3.195(132)$ \\
\hline \multirow{12}{*}{10} & 0.973 & 0.7834 & -6.026 & $0.280(1)$ & $2.416(25)$ & $4.991(108)$ & $4.401(162)$ \\
\hline & 0.971 & 0.8762 & -6.564 & $1.941(2)$ & $2.686(24)$ & $6.397(111)$ & $5.828(134)$ \\
\hline & 0.976 & 0.9460 & -6.835 & $4.145(5)$ & $2.885(37)$ & $7.537(123)$ & $7.066(196)$ \\
\hline & 1.122 & 0.7276 & -5.532 & $0.281(1)$ & $2.247(21)$ & $4.480(101)$ & $3.459(94)$ \\
\hline & 1.117 & 0.8411 & -6.227 & $1.925(2)$ & $2.514(31)$ & $6.118(92)$ & $4.995(153)$ \\
\hline & 1.125 & 0.9191 & -6.554 & $4.154(4)$ & $2.74(28)$ & $7.342(184)$ & $6.260(273)$ \\
\hline & 1.271 & 0.6634 & -4.990 & $0.272(1)$ & $2.098(31)$ & $3.893(53)$ & $2.609(81)$ \\
\hline & 1.275 & 0.8063 & -5.893 & $1.949(2)$ & $2.384(32)$ & $5.853(98)$ & $4.289(143)$ \\
\hline & 1.271 & 0.8940 & -6.294 & $4.166(3)$ & $2.607(25)$ & $7.170(90)$ & $5.542(196)$ \\
\hline & 2.253 & 0.1469 & -1.075 & $0.279(1)$ & $1.631(15)$ & $1.988(23)$ & $0.192(20)$ \\
\hline & 2.243 & 0.6060 & -4.131 & $1.937(3)$ & $1.923(17)$ & $4.583(103)$ & $1.846(67)$ \\
\hline & 2.247 & 0.7478 & -4.886 & $4.161(5)$ & $2.132(25)$ & $6.243(87)$ & $2.985(108)$ \\
\hline \multirow{12}{*}{8} & 1.184 & 0.7831 & -6.854 & $0.356(1)$ & $2.411(31)$ & $4.926(116)$ & $3.821(159)$ \\
\hline & 1.184 & 0.8884 & -7.459 & $2.358(3)$ & $2.634(17)$ & $6.377(74)$ & $4.96(141)$ \\
\hline & 1.176 & 0.9711 & -7.739 & $5.040(6)$ & $2.874(25)$ & $7.535(171)$ & $6.184(195)$ \\
\hline & 1.359 & 0.7272 & -6.323 & $0.332(1)$ & $2.252(25)$ & $4.463(87)$ & $3.069(97)$ \\
\hline & 1.364 & 0.8545 & -7.103 & $2.366(2)$ & $2.517(38)$ & $6.138(122)$ & $4.392(152)$ \\
\hline & 1.358 & 0.9443 & -7.438 & $5.058(4)$ & $2.723(19)$ & $7.381(181)$ & $5.465(133)$ \\
\hline & 1.549 & 0.6652 & -5.746 & $0.345(1)$ & $2.109(21)$ & $3.993(67)$ & $2.392(110)$ \\
\hline & 1.541 & 0.8213 & -6.764 & $2.356(3)$ & $2.406(30)$ & $5.910(99)$ & $3.863(154)$ \\
\hline & 1.544 & 0.9188 & -7.166 & $5.081(3)$ & $2.604(37)$ & $7.234(144)$ & $4.903(151)$ \\
\hline & 2.721 & 0.1479 & -1.267 & $0.333(1)$ & $1.617(11)$ & $2.156(18)$ & $0.189(15)$ \\
\hline & 2.722 & 0.6228 & -4.845 & $2.349(3)$ & $1.942(21)$ & $4.795(84)$ & $1.760(62)$ \\
\hline & 2.726 & 0.7731 & -5.648 & $5.046(5)$ & $2.147(29)$ & $6.448(137)$ & $2.761(90)$ \\
\hline
\end{tabular}


Appendix B:

SAFT-VR Mie EOS results on pure fluids for different $n$.

\begin{tabular}{|c|c|c|c|c|c|c|c|}
\hline$n$ & $T^{*}$ & $\rho^{*}$ & $u^{*}$ & $P^{*}$ & $C_{v}^{*}$ & $\omega^{*}$ & $\gamma_{v}^{*}$ \\
\hline \multirow{12}{*}{12} & 0.856 & 0.788 & -5.635 & 0.248 & 2.597 & 4.481 & 4.077 \\
\hline & 0.851 & 0.883 & -6.110 & 1.747 & 2.665 & 6.036 & 5.894 \\
\hline & 0.852 & 0.952 & -6.354 & 3.713 & 2.728 & 7.397 & 7.442 \\
\hline & 0.985 & 0.733 & -5.195 & 0.256 & 2.440 & 4.084 & 3.450 \\
\hline & 0.985 & 0.847 & -5.804 & 1.735 & 2.534 & 5.797 & 5.127 \\
\hline & 0.987 & 0.925 & -6.111 & 3.725 & 2.616 & 7.232 & 6.641 \\
\hline & 1.120 & 0.667 & -4.681 & 0.259 & 2.297 & 3.627 & 2.687 \\
\hline & 1.119 & 0.812 & -5.502 & 1.728 & 2.427 & 5.555 & 4.461 \\
\hline & 1.118 & 0.898 & -5.877 & 3.706 & 2.525 & 7.041 & 5.954 \\
\hline & 1.960 & 0.141 & -0.889 & 0.246 & 1.632 & 1.933 & 0.190 \\
\hline & 1.977 & 0.597 & -3.728 & 1.733 & 1.999 & 4.340 & 1.927 \\
\hline & 1.968 & 0.745 & -4.523 & 3.706 & 2.158 & 6.076 & 3.217 \\
\hline \multirow{12}{*}{10} & 0.973 & 0.787 & -6.281 & 0.28 & 2.704 & 4.484 & 4.018 \\
\hline & 0.971 & 0.889 & -6.810 & 1.941 & 2.789 & 6.097 & 5.603 \\
\hline & 0.976 & 0.961 & -7.074 & 4.145 & 2.864 & 7.491 & 7.085 \\
\hline & 1.122 & 0.729 & -5.756 & 0.281 & 2.528 & 4.073 & 3.251 \\
\hline & 1.117 & 0.853 & -6.466 & 1.925 & 2.648 & 5.855 & 4.889 \\
\hline & 1.125 & 0.934 & -6.792 & 4.154 & 2.742 & 7.314 & 6.325 \\
\hline & 1.271 & 0.659 & -5.160 & 0.272 & 2.371 & 3.591 & 2.524 \\
\hline & 1.275 & 0.817 & -6.111 & 1.949 & 2.526 & 5.626 & 4.252 \\
\hline & 1.271 & 0.908 & -6.526 & 4.166 & 2.644 & 7.143 & 5.687 \\
\hline & 2.253 & 0.139 & -0.978 & 0.279 & 1.639 & 2.061 & 0.184 \\
\hline & 2.243 & 0.599 & -4.111 & 1.937 & 2.051 & 4.432 & 1.850 \\
\hline & 2.247 & 0.752 & -4.956 & 4.161 & 2.226 & 6.176 & 3.061 \\
\hline \multirow{12}{*}{8} & 1.184 & 0.762 & -7.149 & 0.356 & 2.895 & 4.285 & 3.346 \\
\hline & 1.184 & 0.881 & -7.778 & 2.358 & 2.981 & 6.031 & 4.791 \\
\hline & 1.176 & 0.966 & -8.075 & 5.04 & 3.072 & 7.526 & 6.179 \\
\hline & 1.359 & 0.700 & -6.517 & 0.332 & 2.684 & 3.871 & 2.709 \\
\hline & 1.364 & 0.846 & -7.372 & 2.366 & 2.811 & 5.833 & 4.228 \\
\hline & 1.358 & 0.939 & -7.735 & 5.058 & 2.919 & 7.371 & 5.551 \\
\hline & 1.549 & 0.626 & -5.787 & 0.345 & 2.487 & 3.431 & 2.095 \\
\hline & 1.541 & 0.812 & -6.981 & 2.356 & 2.674 & 5.625 & 3.732 \\
\hline & 1.544 & 0.913 & -7.404 & 5.081 & 2.796 & 7.221 & 5.010 \\
\hline & 2.721 & 0.137 & -1.179 & 0.333 & 1.664 & 2.244 & 0.181 \\
\hline & 2.722 & 0.599 & -4.702 & 2.349 & 2.123 & 4.595 & 1.709 \\
\hline & 2.726 & 0.760 & -5.593 & 5.046 & 2.311 & 6.360 & 2.806 \\
\hline
\end{tabular}




\section{Appendix C:}

MD results on mixture for three molar fractions $x_{1}$. Values in parenthesis represent the error on the last digits (errors on $u^{*}$ and $T^{*}$ are below $0.2 \%$ and $\rho^{*}$ is imposed).

\begin{tabular}{|c|c|c|c|c|c|c|c|}
\hline$x_{1}$ & $T^{*}$ & $\rho^{*}$ & $u^{*}$ & $P^{*}$ & $C_{v}^{*}$ & $\omega^{*}$ & $\gamma_{v}^{*}$ \\
\hline \multirow{12}{*}{0.25} & 0.972 & 0.7712 & -5.739 & $0.268(1)$ & $2.369(31)$ & $4.895(89)$ & $4.352(122)$ \\
\hline & 0.974 & 0.8683 & -6.306 & $1.935(1)$ & $2.632(27)$ & $6.422(112)$ & $5.918(195)$ \\
\hline & 0.974 & 0.9412 & -6.614 & $4.164(4)$ & $2.854(34)$ & $7.637(150)$ & $7.257(208)$ \\
\hline & 1.123 & 0.7105 & -5.217 & $0.280(1)$ & $2.191(19)$ & $4.349(99)$ & $3.286(95)$ \\
\hline & 1.122 & 0.8324 & -5.967 & $1.932(2)$ & $2.469(31)$ & $6.128(132)$ & $5.001(172)$ \\
\hline & 1.123 & 0.913 & -6.327 & $4.160(3)$ & $2.714(27)$ & $7.410(187)$ & $6.444(157)$ \\
\hline & 1.268 & 0.6387 & -4.622 & $0.260(1)$ & $2.057(28)$ & $3.659(69)$ & $2.414(78)$ \\
\hline & 1.273 & 0.7973 & -5.615 & $1.948(1)$ & $2.345(27)$ & $5.849(78)$ & $4.285(141)$ \\
\hline & 1.273 & 0.8862 & -6.036 & $4.157(4)$ & $2.583(33)$ & $7.213(133)$ & $5.670(167)$ \\
\hline & 2.247 & 0.1454 & -1.014 & $0.283(1)$ & $1.625(14)$ & $2.035(25)$ & $0.190(16)$ \\
\hline & 2.244 & 0.5937 & -3.895 & $1.949(2)$ & $1.897(23)$ & $4.557(105)$ & $1.798(42)$ \\
\hline & 2.248 & 0.7380 & -4.667 & $4.169(3)$ & $2.098(19)$ & $6.278(142)$ & $2.956(118)$ \\
\hline \multirow{12}{*}{0.5} & 0.973 & 0.7942 & -6.268 & $0.285(1)$ & $2.454(34)$ & $5.114(111)$ & $4.511(142)$ \\
\hline & 0.972 & 0.8821 & -6.775 & $1.921(2)$ & $2.693(29)$ & $6.436(147)$ & $5.856(145)$ \\
\hline & 0.974 & 0.9524 & -7.051 & $4.15(2)$ & $2.939(30)$ & $7.549(143)$ & $7.206(233)$ \\
\hline & 1.120 & 0.7412 & -5.785 & $0.281(1)$ & $2.260(25)$ & $4.630(87)$ & $3.528(93)$ \\
\hline & 1.118 & 0.8495 & -6.446 & $1.948(2)$ & $2.532(24)$ & $6.196(99)$ & $5.008(162)$ \\
\hline & 1.123 & 0.9267 & -6.773 & $4.191(3)$ & $2.752(39)$ & $7.389(155)$ & $6.230(181)$ \\
\hline & 1.270 & 0.6807 & -5.229 & $0.272(1)$ & $2.143(19)$ & $4.051(63)$ & $2.813(93)$ \\
\hline & 1.271 & 0.8162 & -6.090 & $1.971(2)$ & $2.434(31)$ & $5.904(111)$ & $4.458(97)$ \\
\hline & 1.272 & 0.9004 & -6.475 & $4.171(4)$ & $2.601(31)$ & $7.195(132)$ & $5.454(183)$ \\
\hline & 2.238 & 0.1481 & -1.125 & $0.272(1)$ & $1.640(12)$ & $1.973(28)$ & $0.194(21)$ \\
\hline & 2.250 & 0.6155 & -4.320 & $1.952(2)$ & $1.935(21)$ & $4.658(88)$ & $1.889(53)$ \\
\hline & 2.239 & 0.7555 & -5.088 & $4.152(5)$ & $2.138(33)$ & $6.287(133)$ & $2.998(114)$ \\
\hline \multirow{12}{*}{0.75} & 0.973 & 0.8166 & -6.823 & $0.281(1)$ & $2.542(31)$ & $5.246(83)$ & $4.696(87)$ \\
\hline & 0.976 & 0.8984 & -7.278 & $1.967(2)$ & $2.787(23)$ & $6.449(87)$ & $5.939(195)$ \\
\hline & 0.971 & 0.9667 & -7.539 & $4.161(6)$ & $2.963(32)$ & $7.493(132)$ & $6.935(193)$ \\
\hline & 1.121 & 0.7686 & -6.363 & $0.277(1)$ & $2.358(19)$ & $4.833(67)$ & 3.792(133) \\
\hline & 1.119 & 0.8664 & -6.951 & $1.951(2)$ & $2.609(39)$ & $6.219(99)$ & $5.067(191)$ \\
\hline & 1.123 & 0.9407 & -7.253 & $4.174(4)$ & $2.818(25)$ & $7.313(121)$ & $6.205(202)$ \\
\hline & 1.270 & 0.7165 & -5.840 & $0.274(1)$ & $2.212(20)$ & $4.355(82)$ & $3.054(95)$ \\
\hline & 1.270 & 0.8331 & -6.584 & $1.930(3)$ & $2.459(27)$ & $5.950(132)$ & $4.364(142)$ \\
\hline & 1.272 & 0.9150 & -6.948 & $4.148(5)$ & $2.693(27)$ & $7.121(121)$ & $5.562(188)$ \\
\hline & 2.244 & 0.1510 & -1.248 & $0.262(1)$ & $1.659(11)$ & $1.924(21)$ & $0.199(20)$ \\
\hline & 2.240 & 0.6413 & -4.812 & $1.943(2)$ & $1.986(18)$ & $4.732(43)$ & $2.031(62)$ \\
\hline & 2.239 & 0.7737 & -5.546 & $4.145(5)$ & $2.197(18)$ & $6.267(111)$ & $3.103(96)$ \\
\hline
\end{tabular}


Appendix D:

SAFT-VR Mie EOS results on mixture for three molar fractions (using MX1b).

\begin{tabular}{|c|c|c|c|c|c|c|c|}
\hline$x_{1}$ & $T^{*}$ & $\rho^{*}$ & $u^{*}$ & $P^{*}$ & $C_{v}{ }^{*}$ & $\omega^{*}$ & $\gamma_{v}^{*}$ \\
\hline \multirow{12}{*}{0.25} & 0.972 & 0.777 & -5.862 & 0.268 & 2.618 & 4.316 & 3.793 \\
\hline & 0.974 & 0.874 & -6.422 & 1.935 & 2.701 & 6.019 & 5.444 \\
\hline & 0.974 & 0.951 & -6.707 & 4.164 & 2.782 & 7.478 & 7.011 \\
\hline & 1.123 & 0.718 & -5.328 & 0.28 & 2.445 & 3.889 & 3.018 \\
\hline & 1.122 & 0.838 & -6.087 & 1.932 & 2.567 & 5.782 & 4.737 \\
\hline & 1.123 & 0.923 & -6.436 & 4.16 & 2.666 & 7.295 & 6.246 \\
\hline & 1.268 & 0.651 & -4.705 & 0.26 & 2.293 & 3.363 & 2.275 \\
\hline & 1.273 & 0.803 & -5.757 & 1.948 & 2.456 & 5.559 & 4.129 \\
\hline & 1.273 & 0.896 & -6.173 & 4.157 & 2.570 & 7.112 & 5.586 \\
\hline & 2.247 & 0.153 & -0.925 & 0.283 & 1.630 & 2.080 & 0.183 \\
\hline & 2.244 & 0.602 & -3.840 & 1.949 & 2.009 & 4.409 & 1.782 \\
\hline & 2.248 & 0.740 & -4.674 & 4.169 & 2.178 & 6.173 & 3.001 \\
\hline \multirow{12}{*}{0.5} & 0.973 & 0.788 & -6.499 & 0.285 & 2.801 & 4.461 & 3.969 \\
\hline & 0.972 & 0.887 & -6.999 & 1.921 & 2.869 & 6.016 & 5.459 \\
\hline & 0.974 & 0.960 & -7.261 & 4.15 & 2.936 & 7.415 & 6.925 \\
\hline & 1.120 & 0.733 & -5.986 & 0.281 & 2.612 & 4.079 & 3.256 \\
\hline & 1.118 & 0.854 & -6.663 & 1.948 & 2.717 & 5.827 & 4.824 \\
\hline & 1.123 & 0.935 & -6.979 & 4.191 & 2.803 & 7.274 & 6.231 \\
\hline & 1.270 & 0.667 & -5.403 & 0.272 & 2.446 & 3.632 & 2.570 \\
\hline & 1.271 & 0.820 & -6.319 & 1.971 & 2.589 & 5.622 & 4.240 \\
\hline & 1.272 & 0.908 & -6.703 & 4.171 & 2.693 & 7.095 & 5.596 \\
\hline & 2.238 & 0.139 & -1.037 & 0.272 & 1.654 & 2.036 & 0.185 \\
\hline & 2.250 & 0.605 & -4.289 & 1.952 & 2.082 & 4.457 & 1.875 \\
\hline & 2.239 & 0.755 & -5.132 & 4.152 & 2.255 & 6.171 & 3.071 \\
\hline \multirow{12}{*}{0.75} & 0.973 & 0.806 & -7.147 & 0.281 & 3.002 & 4.544 & 4.049 \\
\hline & 0.976 & 0.898 & -7.596 & 1.967 & 3.046 & 6.029 & 5.418 \\
\hline & 0.971 & 0.969 & -7.845 & 4.161 & 3.107 & 7.351 & 6.789 \\
\hline & 1.121 & 0.755 & -6.641 & 0.277 & 2.788 & 4.209 & 3.405 \\
\hline & 1.119 & 0.866 & -7.248 & 1.951 & 2.873 & 5.834 & 4.824 \\
\hline & 1.123 & 0.943 & -7.540 & 4.174 & 2.947 & 7.210 & 6.125 \\
\hline & 1.270 & 0.698 & -6.095 & 0.274 & 2.609 & 3.832 & 2.793 \\
\hline & 1.270 & 0.832 & -6.888 & 1.93 & 2.727 & 5.616 & 4.257 \\
\hline & 1.272 & 0.918 & -7.250 & 4.148 & 2.822 & 7.043 & 5.533 \\
\hline & 2.244 & 0.140 & -1.157 & 0.262 & 1.681 & 1.997 & 0.187 \\
\hline & 2.240 & 0.626 & -4.800 & 1.943 & 2.168 & 4.510 & 1.984 \\
\hline & 2.239 & 0.769 & -5.611 & 4.145 & 2.336 & 6.166 & 3.118 \\
\hline
\end{tabular}




\section{References}

[1]. I.G. Kaplan, Intermolecular Interactions: Physical Picture, Computational Methods and Model Potentials (John Wiley and Sons, New York, 2006).

[2]. I. Nezbeda, Mol. Phys. 103, 59 (2005).

[3]. T. Young and H. C. Andersen, J. Phys. Chem. B 109, 2985 (2005).

[4]. S. Chapman and T.G. Cowling, The Mathematical Theory of Non-Uniform Gases (Cambridge Mathematical Library, Cambridge, 1970).

[5]. A.Z. Panagiotopoulos, J. Chem. Phys. 112, 7132 (2000).

[6]. H. Okumura and F. Yonezawa, J. Chem. Phys. 113, 9162 (2000).

[7]. I.I. Adamenko, A. N. Grigoriev and Y.I. Kuzovkov, J. Mol. Liq. 105, 261 (2003).

[8]. N.S. Matin and A.H. Jalili, Bull. Chem. Soc. Jap. 78, 2105 (2005).

[9]. G. Galliero, C. Boned, A. Baylaucq and F. Montel, Phys. Rev. E 73, 061201 (2006).

[10]. G. Galliero, C. Boned, A. Baylaucq and F. Montel, Chem. Phys. 333, 219 (2007).

[11]. W.G. Chapman, K.E. Gubbins, G. Jackson, G. and M. Radosz, Fluid Phase Equi. 52, 31 (1989).

[12]. W.G. Chapman, K.E. Gubbins, G. Jackson, G. and M. Radosz, Ind. Eng. Chem. Res. 29, 1709 (1990).

[13]. M.S. Wertheim, J. Stat. Phys. 35, 19 (1984).

[14]. M.S. Wertheim, J. Stat. Phys. 35, 35 (1984).

[15]. M.S. Wertheim, J. Stat. Phys. 42, 459 (1986).

[16]. M.S. Wertheim, J. Stat. Phys. 42, 477 (1986).

[17]. A. Gil-Villegas, A. Galindo, P.J. Whitehead, S.J. Mills, G. Jackson and A.N. Burgess, J. Chem. Phys. 106, 4168 (1997).

[18]. F.J. Blas and L.F. Vega, Mol. Phys. 92, 135 (1997).

[19]. I.G. Economou, Ind. Eng. Chem. Res. 41, 953 (2002). 
[20]. T. Lafitte, D. Bessières, M.M. Piñeiro and J.-L. Daridon, J. Chem. Phys. 124, 024509 (2006).

[21]. T. Lafitte, M.M. Piñeiro, J.-L. Daridon and D. Bessières, J. Phys. Chem. B. 111, 3447 (2007).

[22]. A. Galindo, L.A. Davies, A. Gil-Villegas and G. Jackson, Mol. Phys. 93, 241 (1998).

[23]. M.P. Allen and D.J. Tildesley, Computer simulations of liquids (Oxford Science Publications, Oxford, 1987).

[24]. D. Frenkel and B. Smit, Understanding Molecular Simulation: From Algorithms to Applications (Academic Press, San Diego, 2002).

[25]. J.M. Haile, Molecular Dynamics simulation: Elementary methods (John Wiley \& Sons, New York, 1992).

[26]. T. Boublik, J. Chem. Phys. 53, 471 (1970).

[27]. G.A. Mansoori, N.F. Carnahan, K.E. Starling and T.W. Leland, J. Chem. Phys. 54, $1523(1971)$.

[28]. J.A. Barker and D. Henderson, Rev. Mod. Phys. 48, 587 (1975).

[29]. N.F. Carnahan and K.E. Starling, J. Chem. Phys. 51, 635 (1969).

[30]. J.K. Percus and G.J. Yevick, Phys. Rev. 110, 1 (1958).

[31]. J.P. Hansen and I.R. McDonald, Theory of Simple Liquids (Academic Press, San Diego, 1986).

[32]. B.E. Poling, J.M. Prausnitz and J.P. O'Connell, The properties of Gases and Liquids (McGraw-Hill, New York, 2001).

[33]. H.J.C. Berendsen, J.P.M. Postma, W.F. Van Gusteren, A. Dinola and J.R. Haak, J. Chem. Phys. 81, 3684 (1984).

[34]. J. Kolafa and I. Nezbeda, Fluid Phase Equi. 100, 1 (1994).

[35]. G. Galliero, C. Boned, A. Baylaucq and F. Montel, Fluid Phase Equi. 234, 56 (2005). 
[36]. K. Meier, Ph.D. Thesis, University of the Federal Armed Forces Hamburg, Hamburg, 2002. 
Tables:

Table I : Dimensionless critical properties ${ }^{6}$ used to define reduced quantities.

\begin{tabular}{|c|c|c|c|}
\hline$n$ & $T_{c}{ }^{*}$ & $\rho_{c}{ }^{*}$ & $P_{c}{ }^{*}$ \\
\hline 12 & 1.313 & 0.304 & 0.125 \\
\hline 10 & 1.494 & 0.3 & 0.14 \\
\hline 8 & 1.814 & 0.298 & 0.17 \\
\hline
\end{tabular}

Table II: Comparison of our MD results for a pure LJ fluid with those coming from ${ }^{36}$ for different states.

\begin{tabular}{|c|c|c|c|c|c|}
\hline & $P^{*}$ & $u^{*}$ & $C_{v}{ }^{*}$ & $\omega^{*}$ & $\gamma_{v}{ }^{*}$ \\
\hline AAD (\%) & 0.49 & 0.1 & 0.32 & 0.23 & 0.58 \\
\hline DMax (\%) & 1.47 & 0.32 & 1.66 & 0.64 & 1.51 \\
\hline Bias (\%) & 0.45 & -0.1 & -0.09 & 0.2 & -0.15 \\
\hline
\end{tabular}


Table III: Deviations, eq. (49), between MD and SAFT-VR Mie results for the 12 state points studied and three different repulsive exponents.

\begin{tabular}{|c|c|c|c|c|c|c|}
\hline$n$ & & $u^{*}$ & $\rho^{*}$ & $C_{v}{ }^{*}$ & $\omega^{*}$ & $\gamma^{*}$ \\
\hline \multirow{3}{*}{12} & AAD & 1.23 & 1.14 & 4.00 & 5.97 & 7.88 \\
\cline { 2 - 7 } & DMax & 10.01 & 5.06 & 8.53 & 13.07 & 20.00 \\
\cline { 2 - 8 } & Bias & -0.98 & -0.45 & 1.59 & -5.47 & -7.76 \\
\hline \multirow{3}{*}{10} & AAD & 3.72 & 1.45 & 5.53 & 4.11 & 3.11 \\
\cline { 2 - 8 } & DMax & 8.94 & 5.60 & 13.02 & 10.15 & 8.71 \\
\cline { 2 - 8 } & Bias & 2.16 & 0.23 & 5.41 & -3.49 & -1.99 \\
\hline \multirow{2}{*}{8} & AAD & 3.49 & 2.47 & 11.22 & 5.47 & 4.94 \\
\cline { 2 - 8 } & DMax & 6.88 & 7.04 & 20.09 & 14.09 & 12.44 \\
\hline & Bias & 1.69 & -2.47 & 11.22 & -4.79 & -4.04 \\
\hline
\end{tabular}


Table IV: Deviations, eq. (49), between MD and SAFT-VR Mie results in binary mixtures for the 12 state points studied.

\begin{tabular}{|c|c|c|c|c|c|c|}
\hline$x_{1}$ & & $u^{*}$ & $\rho^{*}$ & $C_{v}{ }^{*}$ & $\omega^{*}$ & $\gamma_{v}^{*}$ \\
\hline \multirow{3}{*}{0.25} & AAD & 2.39 & 1.29 & 4.98 & 4.96 & 4.82 \\
\cline { 2 - 7 } & DMax & 9.57 & 5.04 & 11.62 & 11.84 & 12.85 \\
\cline { 2 - 8 } & Bias & 0.56 & 1.29 & 4.18 & -4.59 & -4.56 \\
\hline \multirow{3}{*}{0.5} & AAD & 3.30 & 1.32 & 6.95 & 5.53 & 4.83 \\
\cline { 2 - 8 } & DMax & 8.50 & 6.12 & 15.58 & 12.77 & 12.01 \\
\cline { 2 - 8 } & Bias & 1.76 & -0.65 & 6.94 & -5.00 & -3.99 \\
\hline \multirow{3}{*}{0.75} & AAD & 3.89 & 1.42 & 9.64 & 5.93 & 5.10 \\
\cline { 2 - 8 } & DMax & 7.84 & 7.52 & 18.23 & 13.39 & -5.02 \\
\hline & Bias & 2.55 & -1.28 & 9.64 & -5.29 & \\
\hline
\end{tabular}


Table V: Deviations, eq. (52), between MD and SAFT-VR Mie + one fluid approximation on $n$, eq.(44), results in binary mixtures for the 12 state points studied.

\begin{tabular}{|c|c|c|c|c|c|c|}
\hline$x_{1}$ & & $u^{*}$ & $\rho^{*}$ & $C_{v}{ }^{*}$ & $\omega^{*}$ & $\gamma_{v}^{*}$ \\
\hline \multirow{3}{*}{0.25} & AAD & 3.27 & 1.41 & 4.93 & 4.14 & 3.13 \\
\cline { 2 - 7 } & DMax & 10.14 & 5.03 & 11.51 & 10.18 & 10.01 \\
\cline { 2 - 8 } & Bias & 1.45 & 0.36 & 4.27 & -3.78 & -2.66 \\
\hline \multirow{3}{*}{0.5} & AAD & 3.74 & 1.35 & 6.64 & 4.87 & 4.04 \\
\cline { 2 - 8 } & DMax & 9.58 & 6.18 & 14.82 & 11.43 & 9.95 \\
\cline { 2 - 8 } & Bias & 2.07 & -0.11 & 6.56 & -4.34 & -2.70 \\
\hline \multirow{3}{*}{0.75} & AAD & 3.89 & 1.37 & 9.00 & 5.61 & 4.72 \\
\cline { 2 - 8 } & DMax & 8.88 & 7.63 & 17.20 & 12.71 & -4.61 \\
\hline & Bias & 2.36 & -1.07 & 9.00 & -4.97 & \\
\hline
\end{tabular}




\section{Figure captions:}

Figure 1: Deviations, eq. (50), on $\rho$ (circles) and $C_{v}$ (triangles) for a given $n(n=10$, grey symbols, $n=8$, open symbols) relatively to the results for $n=12$. a) MD results and b) EOS ones.

Figure 2: Deviations, eq. (50), on $\omega$ (circles) and $\gamma_{v}$ (triangles) for a given $n(n=10$, grey symbols, $n=8$, open symbols) relatively to the results for $n=12$. a) MD results and b) EOS ones.

Figure 3: Deviations, eq. (51), between mixture and one fluid values, a) on $P^{*}$, and b) on $u^{*}$, for different states induced by the various one fluid model: circles, eq (40), down triangles, eq (41), squares, eq (42), diamonds, eq (43) and up triangles, eq (44).

Figure 4: Deviations, eq. (51) between mixture and one fluid values, on $C_{v}{ }^{*}$ (circles), $\omega^{*}$ (triangles) and $\gamma_{v}^{*}$ (square) for different states induced by the various one fluid model. a) eq (40), open symbols, eq (41), black symbols, eq (42), grey symbols; b) eq (43), open symbols, eq (44), grey symbols.

Figure 5: Deviations, eq. $(51)$, a) on $P^{*}$ (circles) and $u^{*}$ (triangles) and b) $C_{v}{ }^{*}$ (circles), $\omega^{*}$ (triangles) and $\gamma_{v}^{*}$ (square), induced by the one fluid model described by eq. (44) for different molar fraction ( $x_{1}=0.25$, open symbols, $x_{1}=0.5$, grey symbols and $x_{1}=0.75$ black symbols). 
Figure 1

a)

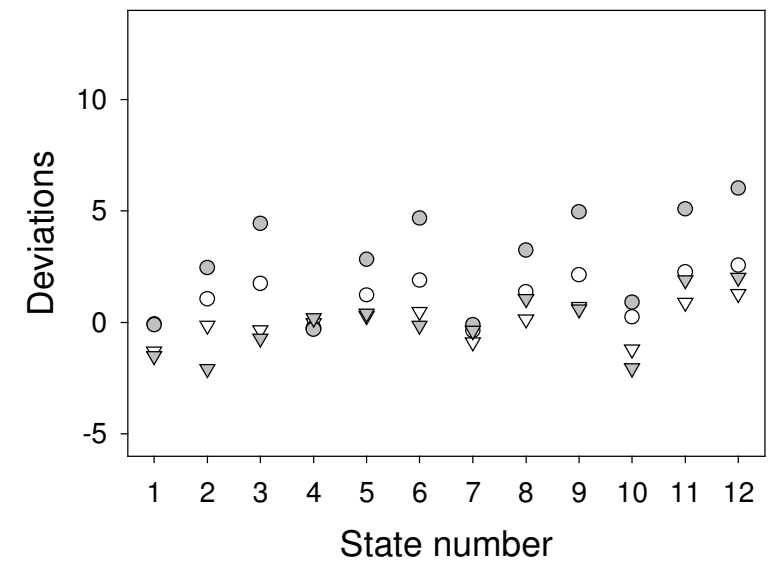

b)

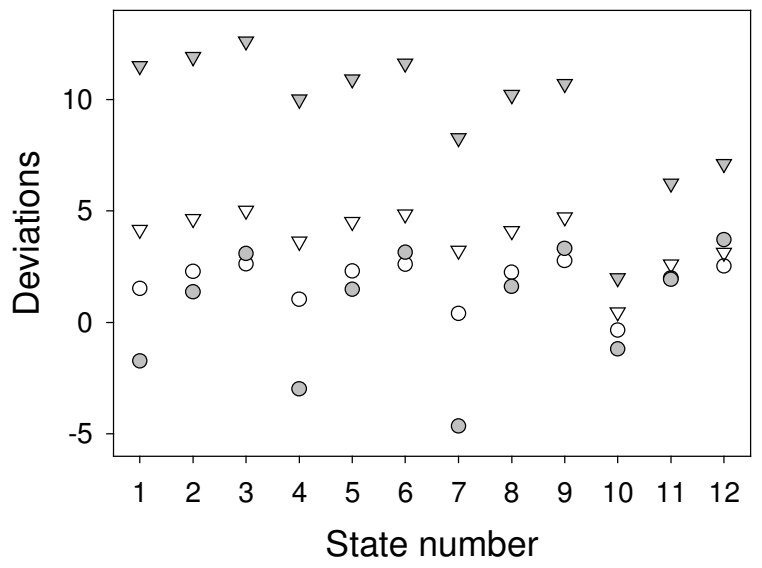




\section{Figure 2}

a)

b)
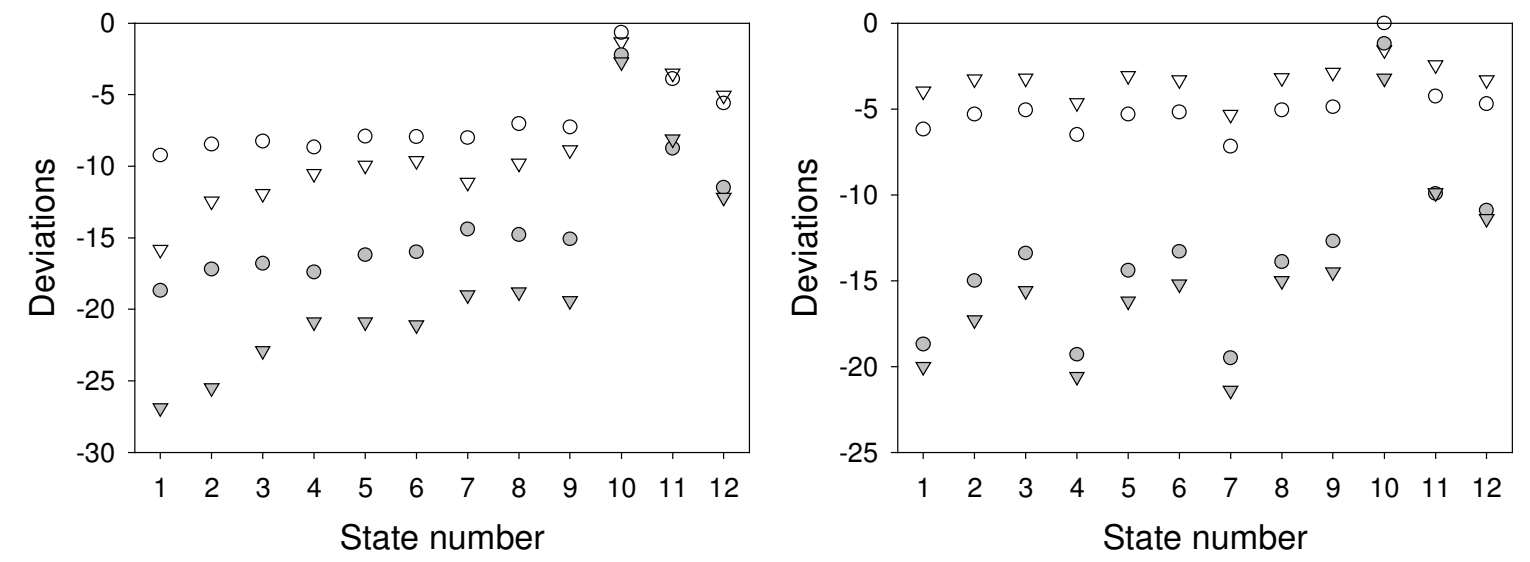
Figure 3

a)

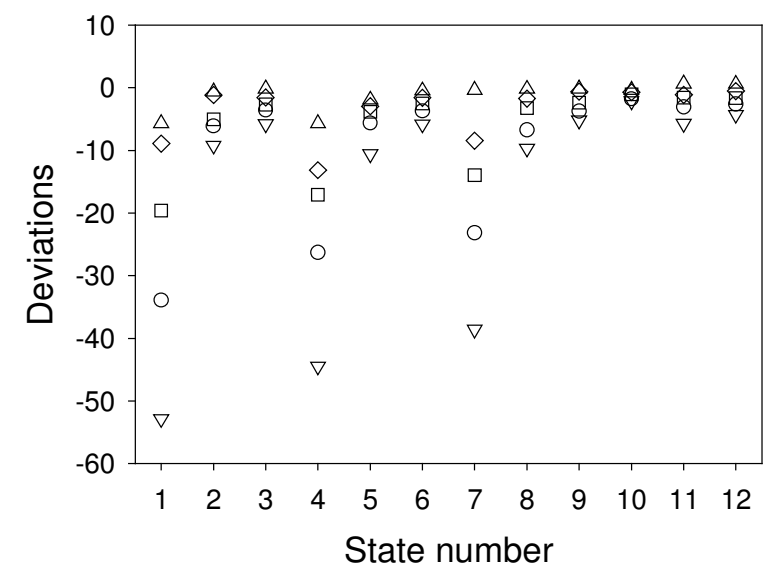

b)

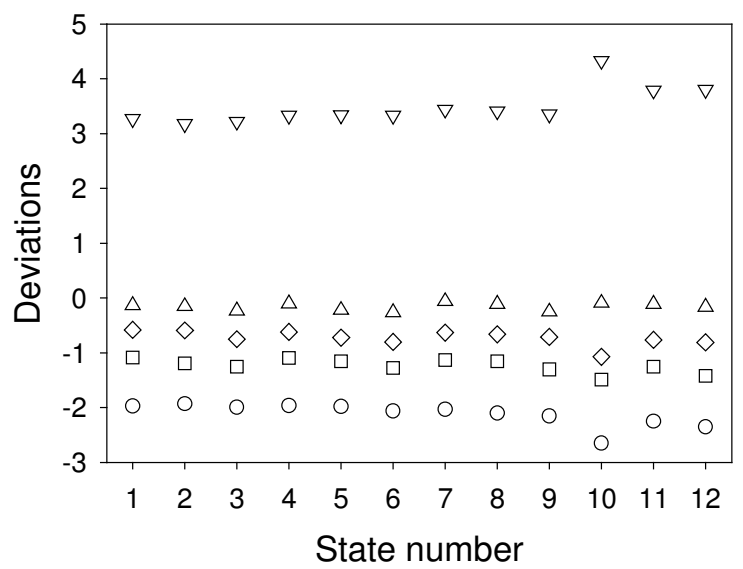


Figure 4

a)

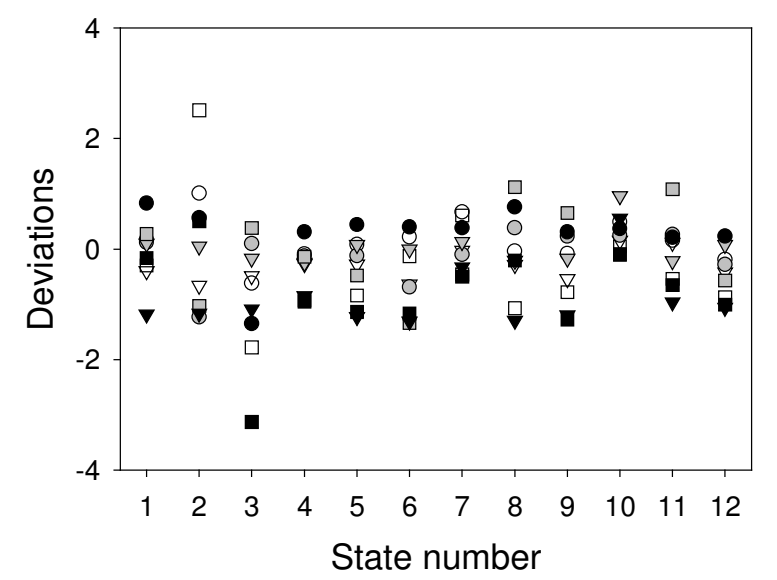

b)

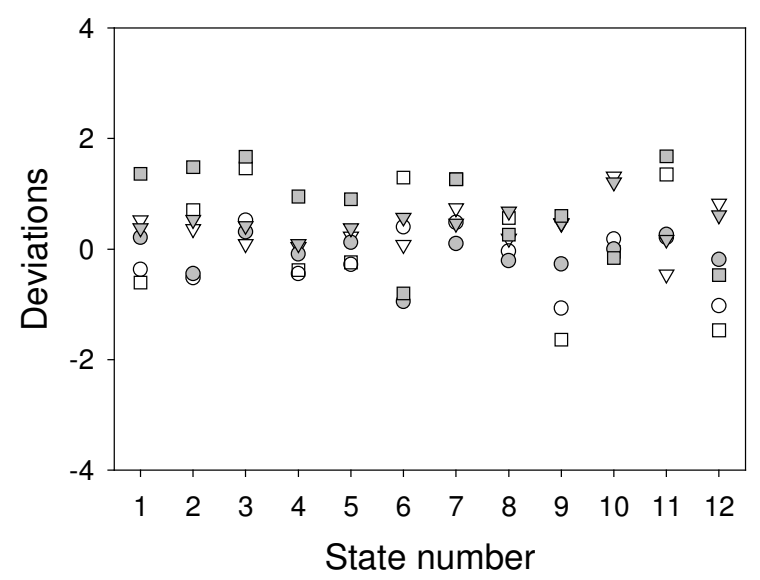


Figure 5

a)

b)
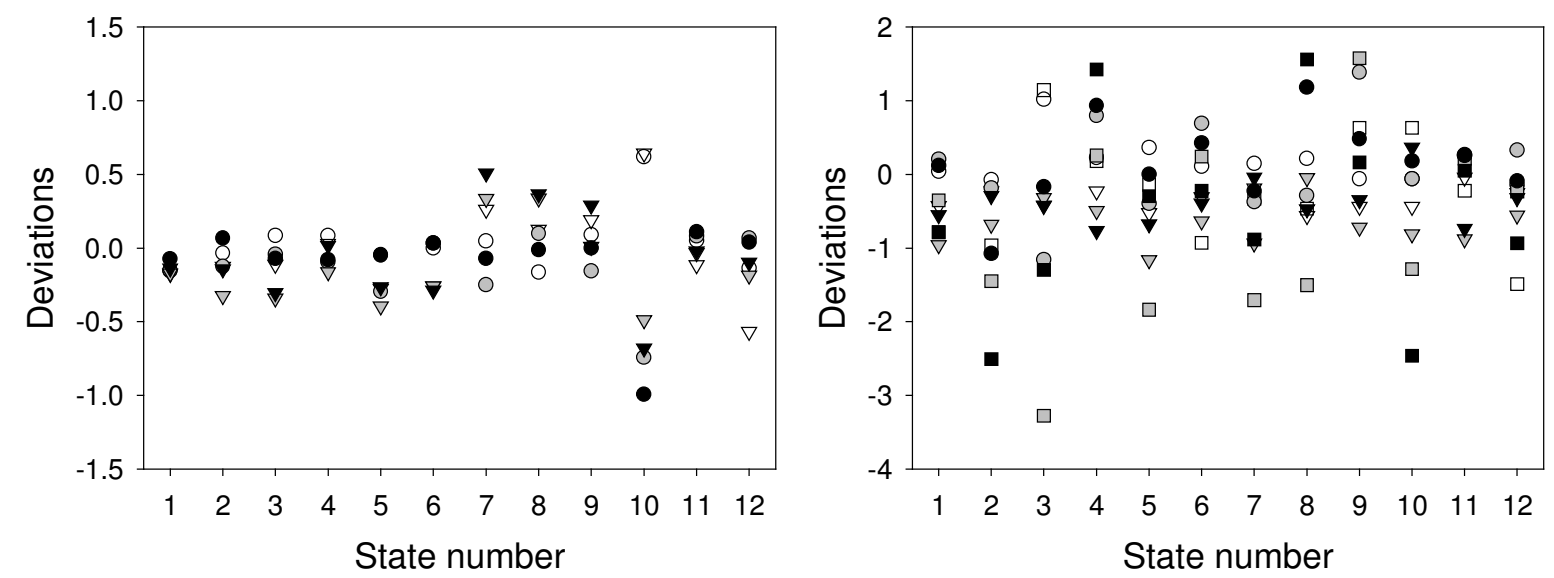